\title{
IMPLANTAÇÃO DE ESCRITÓRIO DE PROJETOS EM INSTITUIÇÃO DE ENSINO SUPERIOR: ESTUDO DE CASO EM UMA UNIVERSIDADE PRIVADA
}

\section{IMPLEMENTATION OF PROJECT OFFICES IN INSTITUTION OF HIGHER EDUCATION: A CASE STUDY}

\section{Eduardo Vimercati}

Doutorando em Administração de Empresas pela Universidade Nove de Julho - UNINOVE.

Professor nos cursos de Administração da Universidade Nove de Julho.

E-mail: eduardovimercatisa@gmail.com

\section{Leandro Alves Patah}

Doutor em Administração pela Universidade Nove de Julho - UNINOVE.

Professor do Mestrado Profissional em Administração da Universidade Nove de Julho.

E-mail: leandro.patah@uol.com.br 


\title{
IMPLANTAÇÃO DE ESCRITÓRIO DE PROJETOS EM INSTITUIÇÃO DE ENSINO SUPERIOR: ESTUDO DE CASO EM UMA UNIVERSIDADE PRIVADA
}

\section{RESUMO}

O presente trabalho traz uma experiência vivida por uma IES (Instituição de Ensino Superior) privada, a partir da qual foi possível apresentar as etapas do projeto de implantação do seu PMO (Project Management Office), as dificuldades e facilidades do processo, bem como as características e atribuições específicas do escritório de gerenciamento de projetos implantado. Além disso, a discussão sobre as particularidades do PMO em uma IES e a apresentação do uso de ferramentas capazes de viabilizar um plano de implantação de PMO em IES possibilitou sua expansão para outras instituições. $O$ trabalho também discute os temas mais relevantes para a literatura, como por exemplo, crescimento das IES e gerenciamento de projetos como ferramenta para otimizar a organização e execução dos diversos projetos gerados nas instituições. Assim, este estudo se propõe a contribuir para a redução de uma lacuna no conhecimento com relação a como implantar PMO em IES, sendo esta a questão de pesquisa deste estudo.

Palavras-chave: escritórios de gerenciamento de projetos; implantação de escritórios de gerenciamento de projetos; instituições de ensino superior.

\section{IMPLEMENTATION OF PROJECT OFFICES IN INSTITUTION OF HIGHER EDUCATION: A CASE STUDY}

\begin{abstract}
This paper presents an experiment conducted by a private HEI (Higher Education Institution), from which it was possible to analyze the implementation stages of its PMO (Project Management Office), the difficulties and facilities, and features and specific tasks of the implemented structure. Moreover, the discussion of the particularities of a PMO in a HEI and the presentation of the use of tools able to allow an implementation plan of the PMO in a HEI, allowed its expansion to other institutions. The paper also discusses the most relevant topics in literature, for example, growth of HEI and project management as a tool to optimize the organization and execution of various projects initiated in the institutions. This study aims to contribute to reducing the knowledge gap in how to implement a PMO in a HEI, which is the research question for this study.
\end{abstract}

Keywords: project management offices; project management office implementation; higher education institutions. 


\section{Introduçãa}

Este estudo pretende investigar em profundidade a implantação dos escritórios de gerenciamento de projetos em instituições de ensino superior, sua gestão e quais métodos são aplicados e desenvolvidos durante este processo. O objetivo é analisar o desenvolvimento de processos conduzidos na perspectiva de se transferir para a sociedade o conhecimento adquirido e desenvolvido em projetos originários nestas instituições.

Um estudo realizado por Siegel, Wadman, Atwater e Link (2004), já mostrava a relevância das universidades no contexto da transferência de tecnologia para as empresas e consequentemente para a sociedade, neste estudo os autores descrevem que as universidades americanas têm sido criticadas por demorar na transferência de novas tecnologias para as empresas e com isso prejudicam o desenvolvimento da competitividade global destas.

Garnica e Torkomian (2009) destacam que a preocupação com o gerenciamento tecnológico no contexto das instituições de ensino superior está diretamente ligada à etapa posterior a atividade de pesquisa que deve resultar em novos conhecimentos para utilização na sociedade. Dessa forma, o primeiro eixo deste estudo será sobre a relevância das instituições de ensino superior para a sociedade, na qual será tratada neste estudo com a sigla IES.

A gestão da tecnologia nas universidades tem se destacado no sistema de inovação brasileiro. A Lei de Inovação de 2004 forneceu diretrizes legais específicas acerca da propriedade intelectual, cooperação técnica e transferência tecnológica favorecendo a intensificação desses processos (Garnica \& Torkomian, 2009).

A cooperação universidade-empresa não apresenta somente o melhoramento tecnológico de produtos ou a criação de inovações, também significa um estreitamento no relacionamento em que a transferência de conhecimento entre as partes é muito importante, pois é por meio dessa transferência que ambas as partes poderão alcançar melhores resultados no processo de pesquisa (Segatto-Mendes, 2001).

É nesse contexto da transferência de conhecimento, tecnologia e interação universidade e empresas que surge a gestão de projetos, por intermédio de uma estrutura capaz de gerenciar os projetos oriundos das instituições de ensino superior (Barbalho; Amaral; Kembichler, Richter \& Torres, 2009).

Segundo o PMBOK, em inglês Project Management Body of Knowledge traduzido para Guia de Conhecimento para o Gerenciamento de Projetos, publicado pelo PMI (2013) Project Management Institute traduzido para Instituto de Gerenciamento de Projeto, os projetos são 
frequentemente utilizados como um meio direto ou indiretamente para atingir os objetivos dentro do plano estratégico de uma empresa.

O primeiro eixo estudado neste trabalho será sobre PMO em IES (Instituições de Ensino Superior), tendo outros termos utilizados, como: EP (Escritório de Projetos) e EGP (Escritório de Gerenciamento de Projetos).

O PMO é uma estrutura de gerenciamento que padroniza os processos de governança relacionados a projetos e facilita o compartilhamento de recursos, metodologias, ferramentas e técnicas. As responsabilidades de um PMO podem variar desde fornecer funções de suporte de gerenciamento de projeto até ser responsável pela gestão direta de um ou mais projetos (PMI, 2013).

Este trabalho também trata de um segundo eixo, que é a implantação de PMO. O atingimento da excelência ou da maturidade em gerenciamento de projetos é mais comprovável com um processo repetitivo que possa ser utilizado em cada um dos projetos. Esse processo repetitivo é chamado de metodologia de gerenciamento de projetos (Kerzner, 2011).

Para Kniess e Moutinho (2012) uma das vantagens da interação universidade-empresa com a implantação dos PMOs é a introdução de uma cultura voltada para projetos e responsável pela gestão deles. Assim, os pesquisadores podem se concentrar mais em aspectos técnicos e científicos.

Para este trabalho são assumidos como pressupostos teóricos a intersecção e relação entre os dois constructos mencionados anteriormente: o PMO em IES e a implantação do PMO. Dessa maneira, foram estudados em profundidade na literatura esses temas, além de pesquisada uma IES, aonde o processo de implantação do PMO vem sendo conduzido.

Diante da identificação, por meio de pesquisa bibliográfica prévia, sobre os temas IES, PMO e Implantação de PMO, no que se refere à implantação de PMO em IES no contexto brasileiro esse processo foi identificado como incipiente, além deste problema a ser explorado, foi observada a inexistência de literatura sobre planos de implantação de PMO em IES, ou seja, não existem estudos de como o processo de implantação de PMO em IES ocorre. 
Com isso, o conteúdo epistemológico deste projeto de pesquisa toma como referência o constructo de IES, suas barreiras e seus pressupostos, PMO, seus tipos e responsabilidades e implantação de PMO.

Diante da lacuna identificada na problemática, ou seja, pouca literatura sobre o processo de implantação de PMO em IES, a questão de pesquisa neste trabalho fica assim definida: Como ocorre o processo de implantação e consolidação de PMO em IES?

Para responder à questão de pesquisa, o objetivo geral deste estudo é analisar a relação entre a unidade de análise IES, PMO, implantação de PMO e propor um plano de implantação de PMO em IES. A partir do objetivo geral, foram definidos os seguintes objetivos específicos:

- analisar como ocorre o processo de implantação do PMO na IES;

- investigar o contex to dos métodos utilizados na implantação de PMO na IES, por meio da disseminação da metodologia de gestão de projetos;

- analisar o alinhamento estratégico entre a instituição e o modelo de PMO adotado.

Para tratar deste tema, este trabalho está estruturado em cinco seções. Esta primeira seção apresenta a introdução ao tema. A segunda traz a revisão da literatura que confere fundamentação teórica à pesquisa. Nela será apresentada o que a literatura acadêmica discorre sobre PMOs em IESs, sua tipologia, características e atribuições, as dimensões que guiam o processo de implantação e as melhores práticas da implantação. A terceira seção, por sua vez, apresentará a descrição metodológica do estudo e se preocupa em estabelecer as formas, os critérios e as normas pelas quais será possível alcançar os resultados obtidos. A pesquisa se propõe a captar e a entender a realidade, para, assim, buscar responder e solucionar um problema e construir novos conhecimentos.

Para isso, será utilizada a pesquisa científica aplicada, com abordagem qualitativa, de natureza exploratória e aplicado o método de estudo de caso único. Após as definições metodológicas, os resultados serão apresentados e analisados na seção de número quatro, para que finalmente na última seção, sejam apresentadas as considerações finais, incluindo limitações e recomendações para a teoria e prática. 


\section{Referencial Teórico}

O referencial teórico deste artigo engloba os seguintes eixos: PMO (Project Management Office - Escritório de Gerenciamento de Projetos) em IES (Instituições de Ensino Superior) e Implantação de PMO.

\section{PMO - Escritório de Gerenciamento de Projetos em IES - Instituições de Ensino Superior}

Em se tratando de IES, sejam elas públicas ou privadas, a Lei $\mathrm{n}^{\circ}$ 9.394, de 20 de dezembro de 1996, conhecida como Lei de Diretrizes e Bases da Educação Nacional (LDB), art. 43, parágrafo III e VI descreve que é essencial o incentivo à pesquisa e a investigação científica com o objetivo do desenvolvimento da ciência e da tecnologia e da criação e disseminação da cultura (Brasil, 1996).

Capes/MEC (2013) apresenta o estado recente e a evolução ao longo dos anos do crescimento das IES privada e pública. Por meio deste estudo nota-se uma tendência de crescimento acentuado, onde se pode observar que o número de instituições de ensino cresceu $72 \%$ em 12 anos, destacando os centros universitários que cresceram $103 \%$ e as faculdades $77 \%$. Esse crescimento torna as instituições de ensino superior um agente relevante para o contexto da sociedade.

Também se faz relevante definir o que são projetos em instituições de ensino superior, visto que essa integração surge a partir dos projetos. Entende-se como extensão o processo educativo, cultural e científico que articula o ensino e a pesquisa de forma indissociável e viabiliza a relação transformadora entre universidade e sociedade (Moraes \& Stal, 1994).

Patah (2004) define o PMO como uma entidade organizacional, tendo como objetivo apoiar os gerentes de projetos e os times da empresa, na implantação dos princípios, práticas, metodologias, ferramentas e técnicas de gestão de projetos.

O PMO é uma estrutura dinâmica criada como solução para a consolidação da área de projetos de uma empresa, com o objetivo de garantir o alcance da estratégia organizacional (Valeriano, 2005). O autor destaca que PMO consiste em uma estrutura formal, instalada em uma empresa e destinada ao apoio à sua área de gerenciamento de projetos proporcionando um intenso elo entre a empresa e seus projetos.

O PMO possui diversas definições, sendo a maioria delas associada ao fato de agregar as fontes das melhores práticas de gerenciamento de projetos. Dessa forma, o PMO é tido como o responsável por implantar, manter e suprir as necessidades da empresa no que se refere a essa disciplina (Crawford, 2002; Kerzner, 2011). 
Segundo o PMI (2013) um PMO é uma estrutura de gestão que padroniza os processos de governança relacionados ao projeto e facilita o compartilhamento de recursos, metodologias, ferramentas e técnicas. As responsabilidades de um PMO incluem oferecer suporte de gerenciamento de projeto para gestão direta de um ou mais projetos.

Valeriano (2005) destaca que as funções do PMO sofrem alterações com o decorrer do tempo e com a maturidade adquirida. Essas atribuições variam também de acordo com a empresa a qual o escritório serve, a depender da natureza, quantidade e complexidade dos projetos.

Para Valeriano (2005) inicialmente, os PMOs se dedicam a:

- controle de prazos e custos;

- elaboração de relatórios multiprojetos e interdepartamentais;

- treinamento em gerenciamento de projetos;

- estabelecimento das relações com os gerentes departamentais;

- melhoria contínua dos processos;

- arquivamento das lições aprendidas.

Mantendo os anteriores, os estágios intermediários são destinados a:

- manutenção do arquivo histórico dos projetos;

- administração dos processos do GP;

- oferecimento de consultoria em projetos;

- desenvolvimento e aperfeiçoamento de métodos e padrões;

- apoio nas avaliações e revisões de projetos.

Nos estágios avançados, também mantidos os anteriores, os PMOs:

- analisam e aprovam propostas de projetos;

- distribuem recursos;

- identificam conflitos e recomendam soluções;

- revisam e avaliam os projetos;

- atuam externamente com foco nos clientes e patrocinadores.

Com maior autoridade ainda, os PMOs focam também: 
- na formação, treinamento e plano de carreira dos gerentes de projetos;

- na gerência direta dos projetos da empresa.

Já segundo o PMI (2013) a função primária de um PMO consiste em apoiar os gestores de projeto em uma variedade de formas as quais podem incluir, mas não estão limitadas a:

- gerenciamento de recursos compartilhados entre todos os projetos administrados pelo PMO;

- identificar e desenvolver metodologia de gerenciamento de projetos, melhores práticas e normas;

- coaching, mentoring, treinamento e supervisão;

- acompanhamento do cumprimento de normas de gerenciamento de projetos, políticas, procedimentos e modelos por meio de auditorias do projeto;

- desenvolvimento e gestão de projetos de políticas, procedimentos, modelos e outras documentações compartilhadas (processo organizacional ativo);

- coordenação das comunicações entre projetos.

Diferentes tipologias de PMOs são propostas na literatura de Aubry e Hobbs (2008); Crawford (2002); Dinsmore e Cabanis-Brewin (2006); Kerzner (2011) e Valeriano (2005); entre outros. Contudo, as identificadas somam um número muito limitado de tipos, geralmente três ou quatro por autor. É difícil de reduzir a grande variedade encontrada de PMOs a limitados tipos classificados na literatura. Estas tipologias não são empiricamente validadas ou sua base empírica não é de domínio público (Aubry \& Hobbs, 2008).

A Figura 1 resume algumas classificações existentes quanto ao tipo de escritórios de projetos e suas respectivas definições. A identificação dos tipos de PMO em IES neste estudo será baseada no modelo criado por Dinsmore, pois é o que mais apresenta tipos de PMO dentre os autores analisados. 


\begin{tabular}{|c|c|c|}
\hline Autor $(a n$ o) & Tipologia & Característic a principal \\
\hline \multirow[t]{3}{*}{$\begin{array}{l}\text { Crawford } \\
\text { (2002); Carvathoe } \\
\text { Rabechini Is. (2011) }\end{array}$} & $\begin{array}{l}\text { Nivel } 1 \text {-Escritório de Con trole da } \\
\text { Proje tos }\end{array}$ & $\begin{array}{l}\text { Ges tầ de grand es a complaxos projetos } \\
\text { individua bente } \\
\text { Foco nos as pect os aden in is tra tivos dos projatos. }\end{array}$ \\
\hline & $\begin{array}{l}\text { Nive1 } 2 \text { - Un idada de Escritório da } \\
\text { Projatos }\end{array}$ & $\begin{array}{l}\text { Integração de todos os projetos no nivelde } \\
\text { unidade de negócios. } \\
\text { Focono gerenciamento da recurs os. }\end{array}$ \\
\hline & $\begin{array}{l}\text { Nivel } 3 \text { - Escritório de Gerenciamento } \\
\text { Es tratég ico de Projetos }\end{array}$ & 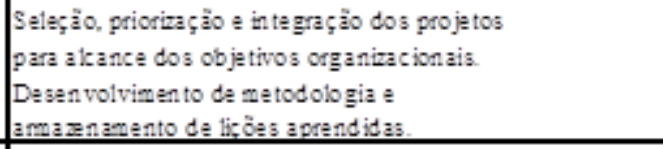 \\
\hline \multirow[t]{5}{*}{$\begin{array}{l}\text { Carvalho e Rabechini Ir } \\
\text { (2011) ad ap tado de } \\
\text { Dinsmore (1998) }\end{array}$} & Equipe Autón ona de Projetos (APT) & 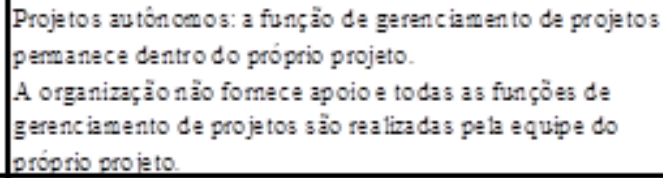 \\
\hline & Escritório de Suporte a os Projetos (PSO) & $\begin{array}{l}\text { Apoio técnico e administrativo às mudanças de } \\
\text { escopo e ao gerencianento de custos. } \\
\text { Responsabilidade pelo sucesso é do gerente da } \\
\text { projato. }\end{array}$ \\
\hline & $\begin{array}{l}\text { Centro de Excelancia de Gerenciamento } \\
\text { de Proje tos (PMCOE) }\end{array}$ & 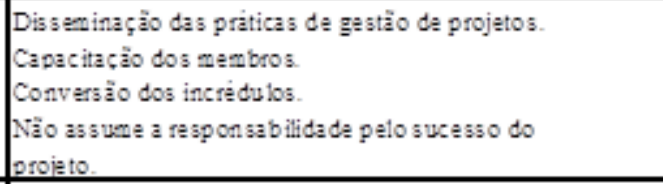 \\
\hline & $\begin{array}{l}\text { Es critório da Gerenciamento da } \\
\text { Programas (PrgMo) }\end{array}$ & $\begin{array}{l}\text { Compreende as funçöes do PMCOE e, en } \\
\text { alguns casos, as do PSO. } \\
\text { Seu funcionamento depende de poder, prioridade } \\
\text { corporativa a controle. } \\
\text { As sume res pons abilidade pelo sucesso do } \\
\text { projato. }\end{array}$ \\
\hline & Lider de Proieto (CPO) & $\begin{array}{l}\text { Gerencia o portfólio de proje tos. } \\
\text { A tua no planejanento estrategico. } \\
\text { Implementa e avalia os projetos estratégicos. } \\
\text { Gerencia stakeholders de alto nivel. }\end{array}$ \\
\hline \multirow[t]{3}{*}{ Kermer (2011) } & PO (Escritório de Projetos) Funciona1 & Gerenciarracurs os criticos. \\
\hline & PO de Grupo do Cliente & $\begin{array}{l}\text { Gerenciamento do cliente e comun icação } \\
\text { coen ocliente. }\end{array}$ \\
\hline & PO Corpora tivo (ou Es traté gico) & $\begin{array}{l}\text { Centra-se en quastôes corporativas a } \\
\text { estrateggicas }\end{array}$ \\
\hline \multirow[t]{2}{*}{ Valeriano (2005) } & PMO quanto a Atribuiçōes & $\begin{array}{l}\text { Apoio a proje tos; treinamento; consultoria } \\
\text { métod os a padrobs; gerencia de projatos. }\end{array}$ \\
\hline & PNO quanto à Forma & Estrutura fisica. virtual e mista. \\
\hline \multirow[t]{2}{*}{$\begin{array}{l}\text { Descuza e } \\
\text { Evaris to }(2006)\end{array}$} & Dimens åo a derinis trativa & $\begin{array}{l}\text { A po io aden in is trativo. } \\
\text { Focono gerenciamento das tarefas, recursos a } \\
\text { afins. }\end{array}$ \\
\hline & $\begin{array}{l}\text { Dimens ào de conhecinento } \\
\text { intensivo }\end{array}$ & 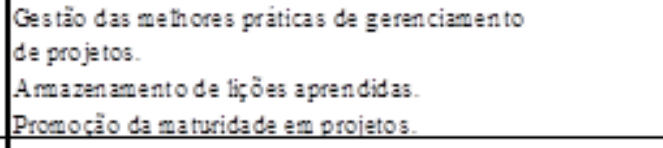 \\
\hline \multirow{3}{*}{ PMBBOK (2013) } & Apoio: & 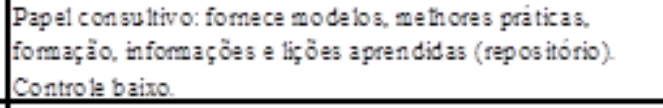 \\
\hline & Controlad or: & $\begin{array}{l}\text { Controlam a través de vários meios, adoção de estruturas } \\
\text { de gerenciamento de projetos ou metodologias. } \\
\text { Controla moderado. }\end{array}$ \\
\hline & Direcionad or: & $\begin{array}{l}\text { Assumen por compla to o PMO } \\
\text { Controla alto. }\end{array}$ \\
\hline
\end{tabular}

Figura 1. Classificações dos tipos de escritórios de projetos Fonte: Elaborada pelos autores 


\section{Implantação de PMO}

Para Abe e Carvalho (2005), as formas de estruturação dos escritórios de projetos, sua importância nas empresas e suas responsabilidades, podem variar de empresa para empresa, baseando-se nas quantidades e capacidade dos projetos que possuem, em determinado período de tempo.

A implantação de um PMO requisita investimento para dar suporte e colocar em prática os projetos. A aposta é que o retorno do dinheiro e tempo investido no PMO traga melhores resultados, como maior rapidez e menor custo para cada projeto individualmente. O valor agregado está na implantação de processos, práticas e padronização, que capacitarão todos os projetos dentro da empresa a atingir suas expectativas (Abe \& Carvalho, 2005).

Segundo Carvalho e Rabechini Jr. (2011) o processo de implantação deve iniciar com a formação dos fundamentos do PMO e o desenvolvimento de uma metodologia de gestão de projetos para a empresa. Na próxima etapa, deve-se identificar e selecionar os projetos com maior prioridade, identificando projetos-piloto para a aplicação da metodologia de GP, treinando e capacitando suas equipes.

Com relação à instalação de um PMO, Valeriano (2005) descreve que para atingir a maturidade, um PMO passará por quatro processos: projeto, implantação, ampliação e melhoria contínua.

Um estudo realizado por Barbalho et al. (2009) mostra que a implantação progressiva de um PMO traz menor tensão nos resultados obtidos durante esse processo. Portanto, desde que implantadas de maneira criteriosa, as técnicas de gerenciamento de projetos podem trazer benefícios significativos, mesmo em pequenas e médias empresas de alta tecnologia. Para isso, entretanto precisam ser implantadas de forma a equilibrar as demandas não somente dos gerentes, mas de todos os envolvidos nos projetos.

Coelho, Dubke e Nascimento (2010) observam que a importância do PMO na empresa ao longo do tempo se faz cada vez mais necessária e permanente, sem o caráter temporário dos projetos. O PMO tem uma estrutura central, com a finalidade de integrar processos, ferramentas e indicadores, e serve como base para os profissionais e as competências da empresa.

Patah e Carvalho (2003) descrevem alguns fatores críticos na implantação de um PMO em uma empresa. Os autores destacam que o primeiro fator crítico é o patrocínio e apoio da alta direção, e concluem que sem esse apoio o projeto de implantar um PMO pode terminar mesmo 
antes de ter iniciado. O segundo fator crítico é a realização de uma avaliação da estrutura da empresa e dos tipos de projetos que esta empresa executa, pois isso trará consonância do tipo de PMO com a missão, os objetivos e os benefícios que a empresa espera. O terceiro fator crítico citado pelos autores é o alinhamento estratégico entre o PMO e a empresa, com o objetivo de executar somente as mudanças necessárias à empresa. Por fim, o quarto fator crítico refere-se à integração da implantação do PMO com os sistemas de informação da empresa e seus processos.

A Figura 2, a seguir, traz um quadro comparativo dos FCS pesquisados neste trabalho. A relação dos FCS está ligada diretamente com a implantação e o desempenho do PMO, pois sem o apoio da alta gestão, o alinhamento estratégico e a comunicação eficiente fica claro que seria muito difícil o sucesso da implantação. 
Implantação de Escritório de Projetos em Instituição de Ensino Superior: Estudo de Caso em uma Universidade Privada

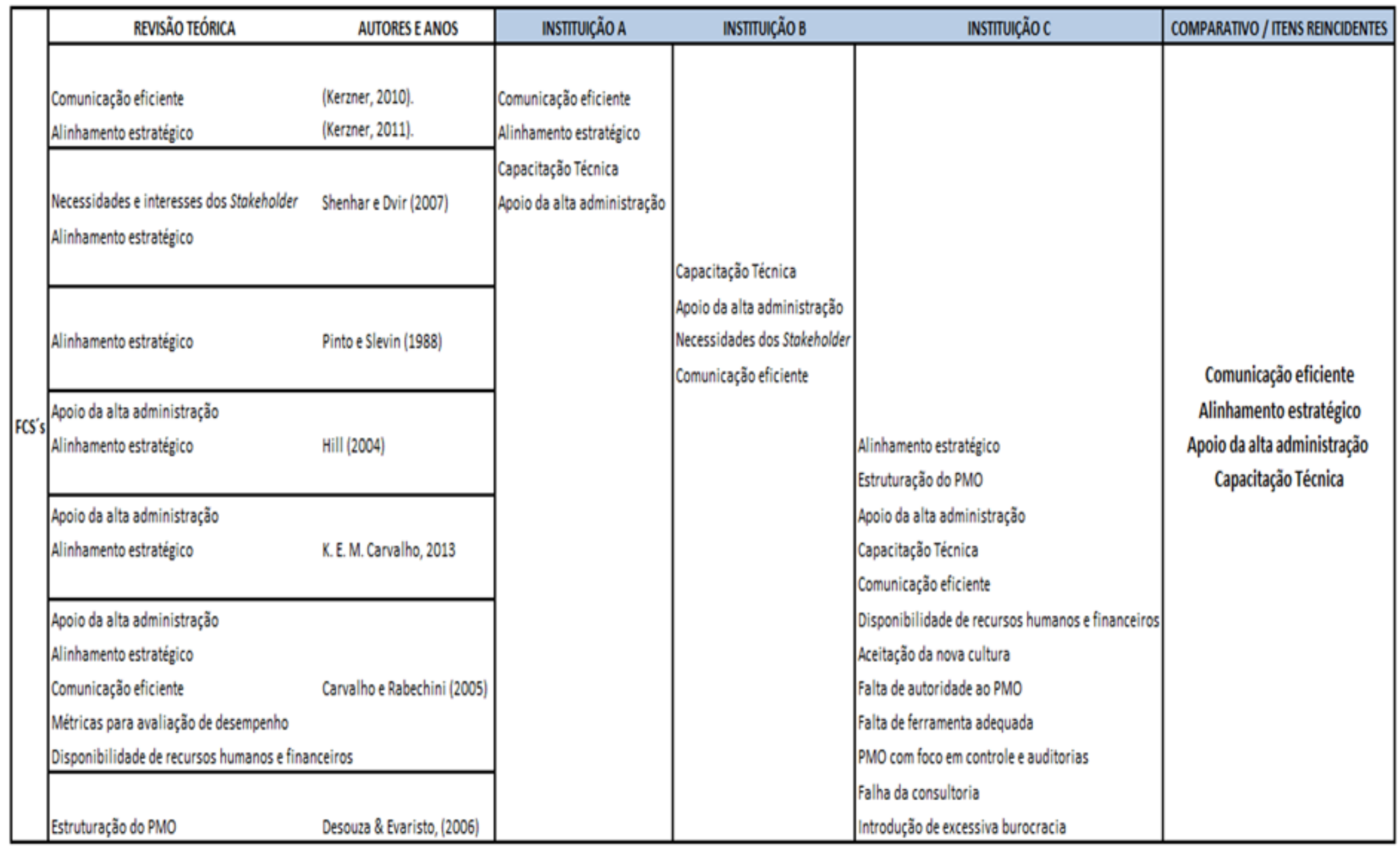

Figura 2. Classificações dos tipos de escritórios de projetos

Fonte: Elaborada pelos autores.

Revista de Gestão e Secretariado - GeSec, São Paulo, v. 7, n. 1, p. 85-111,jan./abr. 2016. 


\section{METODOLOGIA}

O presente estudo caracterizou-se como qualitativo, exploratório e utilizando o método de estudo de caso, em que foi feita a triangulação das seguintes técnicas: entrevistas, pesquisa bibliográfica (artigos, folders, sites, dissertações) e observação direta. Quanto às entrevistas, estas foram realizadas com o responsável pelo PMO e professores envolvidos em projetos da IES junto aos programas stricto sensu, sendo que o responsável pelo PMO contou com a colaboração de aproximadamente 15 professores do programa de mestrado profissional em administração em gestão de projetos durante o processo de implantação. O tempo estimado de duração das entrevistas foi de 30 horas somando-se todos os envolvidos. A coleta de dados se deu por meio de documentos, templates, apresentações e protocolos criados para a organização dos projetos da IES. E por fim, a observação direta se deu por meio de participação de reuniões sobre o processo de organização dos projetos e de treinamentos para os envolvidos.

A investigação do estudo de caso, essencialmente compreende um método abrangente englobando a lógica do projeto, as técnicas de coleta de dados e as abordagens específicas à análise de dados, contando com múltiplas fontes de evidência, convergindo dados de forma triangular beneficiando-se do desenvolvimento de teorias anteriores como forma de orientação de coleta e análise de dados (YIN, 2010).

O estudo de caso único, justifica-se nesta pesquisa por mostrar-se crítico, extremo, representativo, revelador e longitudinal em sua essência, abrangência e totalidade.

Os métodos e técnicas a serem empregados na pesquisa científica podem ser selecionados desde a proposição do problema, da formulação das hipóteses e da delimitação do universo ou da amostra. A seleção do instrumental metodológico está, portanto, diretamente relacionada com o problema a ser estudado, e a escolha dependerá dos vários fatores relacionados com a pesquisa, ou seja, a natureza dos fenômenos, o objeto da pesquisa, os recursos financeiros, a equipe humana e outros elementos que possam surgir no campo da investigação (Marconi \& Lakatos, 2005).

Segundo Yin (2010), o estudo de caso é uma investigação empírica que investiga um fenômeno contemporâneo em profundidade e em seu contexto de vida real, especialmente quando os limites entre fenômeno e o contexto não são claramente evidentes.

A opção deste trabalho, por um estudo de caso único, deu-se pelo fato de a organização estar, quando do início desta pesquisa, justamente em um processo de implementação de um PMO, o que possibilitou ao pesquisador obter informações da IES antes, durante e na fase final de implantação 
da estrutura, com amplo acesso à organização e às informações. Isso levou a caracterizar o caso como caso incomum ou mesmo raro, como visto anteriormente.

Gil (2008) define que o estudo de caso é caracterizado pelo estudo aprofundado e completo de um ou poucos objetos, permitindo seu conhecimento amplo e detalhado, tarefa praticamente impossível mediante outros tipos de delineamentos.

A Figura 3 representa as etapas deste estudo, sendo elas: a implantação de PMO na IES; a coleta de dados; análise dos dados e a proposta de um plano para implantação de PMO.

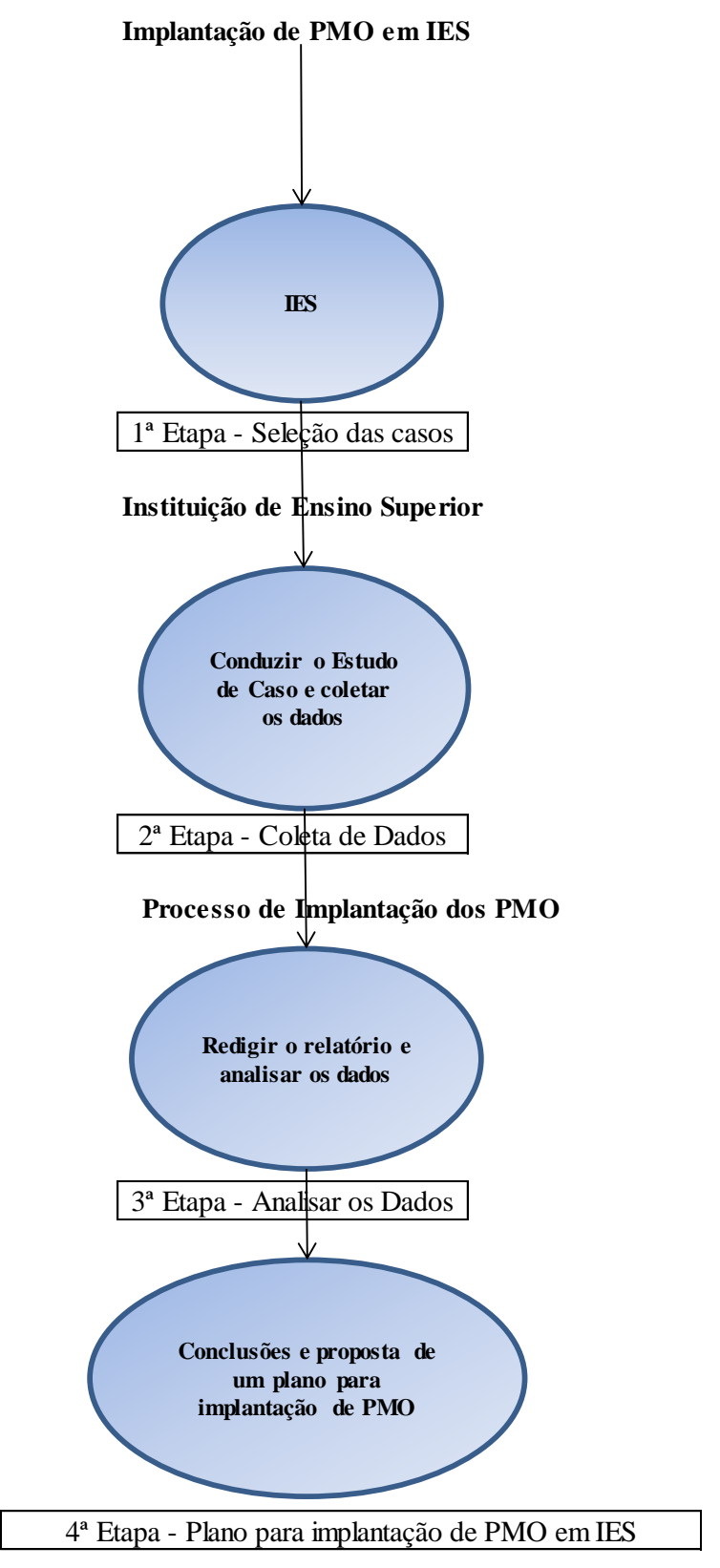

Figura 3. Classificações dos tipos de escritórios de projetos

Fonte: Elaborada pelos autores 
O estudo de caso foi realizado em quatro etapas: a primeira, que consistiu na seleção do caso em questão devido à relevância quanto às metodologias utilizadas para implantação do PMO na IES e ao momento em que a instituição se encontrava no início de implementação da estrutura. Tal instituição está localizada no estado de São Paulo. A segunda etapa envolveu a condução do estudo de caso e a coleta dos dados na instituição, por intermédio de um roteiro de entrevista conforme apresentado na sequência, juntamente com pesquisa documental e análise de conteúdo. A terceira etapa consistiu na redação do relatório do caso e na análise dos dados. A quarta e última etapa tratou das conclusões a serem obtidas do caso, na modificação da teoria, no desenvolvimento das implicações teóricas e na elaboração de um relatório dos casos cruzados. Após o fechamento do caso, foi elaborado e proposto um plano para implantação de PMO em IES.

A operacionalização das variáveis e proposições está organizada, na Figura 4, a partir dos objetivos específicos e das categorias determinadas com base na revisão da literatura conduzida na seção 2, relacionando cada proposição aos respectivos autores.

\begin{tabular}{|c|c|c|c|}
\hline Objetivos & Variáveis & Proposições & Autores (ano) \\
\hline \multirow{3}{*}{1} & \multirow{3}{*}{$\begin{array}{l}\text { Como ocorre o processo } \\
\text { de implantação do PMO } \\
\text { em IES }\end{array}$} & $\begin{array}{l}\text { V. 1: A primeira fase da implantação de um PMO é a decisão } \\
\text { de tê-la ou não em sua organização. }\end{array}$ & Abe e Carvalho (2005) \\
\hline & & $\begin{array}{l}\text { V. 2: A instalação de um PMO deve ser objeto de um projeto } \\
\text { específico. }\end{array}$ & Valeriano (2005) \\
\hline & & $\begin{array}{l}\text { V. 3: A identificação do motivo que fundamenta a existência } \\
\text { do PMO, a elaboração do seu planejamento estratégico e a } \\
\text { definição das métricas para avaliação de desempenho são } \\
\text { fatores críticos de sucesso na implantação do PMO. }\end{array}$ & Desouza e Evaristo (2006) \\
\hline \multirow[t]{2}{*}{3} & \multirow[t]{2}{*}{$\begin{array}{l}\text { Alinhamento estratégico } \\
\text { entre o PMO e a IES }\end{array}$} & $\begin{array}{l}\text { V. 4: O alinhamento estratégico entre a organização e o PMO } \\
\text { é fator decisivo de sucesso na introdução de uma estrutura } \\
\text { de gestão de projetos. }\end{array}$ & $\begin{array}{l}\text { Aubry et al. (2010); } \\
\text { Carvalho e Rabechini (2005); } \\
\text { Desouza e Evaristo (2006); } \\
\text { Kerzner (2011). } \\
\end{array}$ \\
\hline & & $\begin{array}{l}\text { V. 5: A alta administração precisa reconhecer o valor e o impacto } \\
\text { do gerenciamento formal de projetos no desempenho institucio- } \\
\text { nal. }\end{array}$ & $\begin{array}{l}\text { Anselmo e Maximiano (2003); } \\
\text { Carvalho e Rabechini (2005); } \\
\text { Desouza e Evaristo (2006. }\end{array}$ \\
\hline \multirow{5}{*}{4} & \multirow{5}{*}{$\begin{array}{l}\text { Características e atribuições do } \\
\text { PMO nas IES }\end{array}$} & $\begin{array}{l}\text { V. 6: O PMO é responsável pelo desenvolvimento de um conjunto } \\
\text { de normas e regras que representam a metodologia própria da } \\
\text { organização para a gestão dos seus projetos, padronizando os } \\
\text { processos. }\end{array}$ & $\begin{array}{l}\text { Andersen et al. (2007); } \\
\text { Daí e Wells (2004); } \\
\text { Hobbs e Aubry (2007); } \\
\text { Valeriano (2005) }\end{array}$ \\
\hline & & $\begin{array}{l}\text { V. 7: O PMO é responsável pela coleta e pelo armazenamento } \\
\text { sistemático do conhecimento para transformá-lo nas lições apren- } \\
\text { didas, aprimorando a metodologia utilizada e conferindo maturida- } \\
\text { de para a instituição. }\end{array}$ & $\begin{array}{l}\text { Daí e Wells (2004); } \\
\text { Valeriano (2005) }\end{array}$ \\
\hline & & $\begin{array}{l}\text { V. 8: O PMO promove a melhoria contínua dos processos de gestão } \\
\text { de projetos. }\end{array}$ & Valeriano (2005) \\
\hline & & $\begin{array}{l}\text { V. 9: O PMO é responsável pelo treinamento da equipe e desenvol- } \\
\text { vimento de competências em gestão de projetos. }\end{array}$ & $\begin{array}{l}\text { Hobbs e Aubry (2007); } \\
\text { Valeriano (2005) }\end{array}$ \\
\hline & & $\begin{array}{l}\text { V. 10: O PMO promove o aprendizado organizacional, a inovação e } \\
\text { a gestão do conhecimento. }\end{array}$ & $\begin{array}{l}\text { Hobbs e Aubry (2007); } \\
\text { Aubry et al. (2010); }\end{array}$ \\
\hline \multirow{3}{*}{2} & \multirow{3}{*}{$\begin{array}{l}\text { Disseminação da metodologia } \\
\text { de gerenciamento de projetos. }\end{array}$} & $\begin{array}{l}\text { V. 13: O PMO do ponto de vista organizacional, pode ser visto como } \\
\text { uma ferramenta de apoio à gestão do conhecimento nas instituições. }\end{array}$ & $\begin{array}{l}\text { Carvalho e Nadae (2012); } \\
\text { Kawahara e Jussani (2013). }\end{array}$ \\
\hline & & $\begin{array}{l}\text { V. 14: O PMO é responsável pelo desenvolvimento de competências } \\
\text { e metodologias de gerenciamento de projetos. }\end{array}$ & $\begin{array}{l}\text { Hobbs e Aubry (2010); } \\
\text { PMI (2013). }\end{array}$ \\
\hline & & $\begin{array}{l}\text { V. 15: Seleção, priorização e integração dos projetos para alcance dos } \\
\text { objetivos organizacionais, desenvolvimento de metodologia e armaze- } \\
\text { namento de lições aprendidas. }\end{array}$ & $\begin{array}{l}\text { Crawford (2002); } \\
\text { PMI (2013). }\end{array}$ \\
\hline
\end{tabular}




\begin{tabular}{|c|c|c|c|}
\hline \multirow{2}{*}{5} & \multirow{2}{*}{$\begin{array}{l}\text { Transferência de conhecimento } \\
\text { na implantação de um PMO. }\end{array}$} & $\begin{array}{l}\text { V. 16: O mercado encontra-se cada vez mais competitivo e exigindo } \\
\text { rapidez e assertividade nos projetos desenvolvidos nas organizações. }\end{array}$ & $\begin{array}{l}\text { Coelho et al. (2010); } \\
\text { Martins et al. (2005); } \\
\text { Crawford (2002). }\end{array}$ \\
\hline & & $\begin{array}{l}\text { V. 17: A agressiva competição do mercado globalizado pressiona as } \\
\text { organizações a buscarem formas de superar as dificuldades e garantir } \\
\text { sua sobrevivência. }\end{array}$ & Carvalho e Abe (2006); \\
\hline \multirow{5}{*}{5} & \multirow{5}{*}{$\begin{array}{l}\text { Relação entre universidade e } \\
\text { empresas. }\end{array}$} & $\begin{array}{l}\text { V. 18: A universidade tem como foco principal o investimento na gera- } \\
\text { ção de conhecimentos. }\end{array}$ & Moraes e Stal (1994); \\
\hline & & $\begin{array}{l}\text { V. 19: As empresas têm interesse em pesquisas aplicadas a temas } \\
\text { específicos e na resolução de problemas próprios. }\end{array}$ & Moraes e Stal (1994); \\
\hline & & $\begin{array}{l}\text { V. 20: A aproximação entre universidades e empresas tem sido uma } \\
\text { forma encontrada por vários países para adequar os recursos humanos } \\
\text { formados à realidade do mercado. }\end{array}$ & $\begin{array}{l}\text { Lima e Teixeira (2001); } \\
\text { Porto (2004). }\end{array}$ \\
\hline & & $\begin{array}{l}\text { V. 21: A percepção da universidade, enquanto agente de desenvolvi- } \\
\text { mento ecônomico. }\end{array}$ & $\begin{array}{l}\text { Segatto-Mendes e Mendes } \\
\text { (2006); } \\
\text { Segatto (1996). }\end{array}$ \\
\hline & & $\begin{array}{l}\text { V. 22: As relações entre universidades e empresas possibilitam a melhor } \\
\text { difusão e transferência de novos conhecimentos e tecnologia. }\end{array}$ & $\begin{array}{l}\text { Takahashi e Barrenha (2010); } \\
\text { Porto et al. (2010). }\end{array}$ \\
\hline
\end{tabular}

Figura 4. Operacionalização das variáveis e preposições

Fonte: Elaborada pelos autores

A escolha da unidade de análise baseou-se em pesquisa na literatura, $\mathrm{n}$ qual foram identificadas poucas IES que fizeram ou estavam em processo de implantação de um PMO. Outro fator relevante é a facilidade de acesso por motivos de contato com profissionais envolvidos diretamente no processo de implantação. Também foram utilizados alguns critérios para a escolha da instituição pesquisada, sendo eles:

- possibilidade de ação e intervenção no contexto organizacional: interesse dos envolvidos em contribuir para comprovação empírica da teoria apresentada e no desenvolvimento futuro das habilidades profissionais em outras instituições;

- confiabilidade das informações: quanto mais confiáveis e menos ocultas as informações, maior a contribuição para o estudo e, consequentemente, maior a possibilidade de aplicação dos resultados;

- facilidade de acesso: contato com profissionais envolvidos diretamente no processo de implantação do PMO.

A IES está localizada no estado de São Paulo. A unidade de análise é o próprio PMO Project Management Office ou Escritório de gerenciamento de projetos. Os projetos a serem selecionados são oriundos do departamento de pós-graduação em administração da instituição. 
Por se tratar de uma IES privada que se encontrava em processo de início de implantação com total apoio da alta gestão da instituição, considerou-se a relevância deste tipo de organização. Também deve-se levar em conta o fato de que poucas IES implantaram uma estrutura como PMO. Essa afirmação foi corroborada pela pesquisa na literatura nacional. Outra justificativa é a própria importância dentro do contexto de instituições de ensino superior do raro fato de tais organizações implantarem uma estrutura (PMO) normalmente voltada para organizações ou empresas de cunho comercial.

\section{Apresentação e Análise de Resultados}

A instituição objeto desta pesquisa é uma instituição privada de ensino superior, fundada nos anos 1970, e que conta hoje com mais de 100.000 alunos, distribuídos em mais de 100 cursos de Graduação e Pós-Graduação, possuindo campi localizados na cidade de São Paulo e no interior do estado de São Paulo.

A instituição oferece as seguintes modalidades de ensino: colégio (educação infantil, ensino fundamental I, ensino fundamental II, ensino médio e técnicos profissionalizantes), cursos de graduação e curta duração, especialização e MBA lato sensu e pós-graduação stricto sensu, além de cursos de extensão. Na Pós-graduação stricto sensu há 21 cursos nas áreas de ciências da saúde, humanas, exatas, sociais aplicadas e direito.

A unidade de análise desta pesquisa é o escritório de gerenciamento de projetos ou PMO que está localizado junto ao programa de pós-graduação em administração dentro de ciências sociais aplicadas.

\section{Informações Coletadas}

O PMO da instituição foi instituído em 2013 com a seguinte missão: desenvolver e difundir as práticas profissionais em gestão de projetos. A visão da estrutura compreende possibilitar que o PMO atue como órgão chancelador de projetos técnicos da universidade para programas de mestrados profissionais, tornar o PMO o meio-campo pulsante entre a universidade, os profissionais e as empresas e focar a gestão da produção acadêmica e a aprovação dos projetos de inovação.

A área foi criada com os seguintes objetivos: 
- prestar serviços de apoio ao ensino e a pesquisa em temas relacionados a gerenciamento de projetos desenvolvidos no âmbito de estudos acadêmicos, técnicos e de inovação tecnológica (Pesquisa, Desenvolvimento e Inovação - PDI);

- realizar a prospecção, a disseminação e prover auxílio no desenvolvimento e utilização de metodologias orientadas a gestão de projetos, tendo em vista seu relacionamento com as atividades de ensino, pesquisa e extensão;

- induzir e difundir o uso de um conjunto de ferramentas informacionais pautando-se pelo ensino e sistematização de seu emprego.

E tem como principais atividades:

- estabelecer a metodologia de gerenciamento de projetos de inovação tecnológica do programa incluindo:

- o fluxo dos projetos com processos e fases e duração de cada etapa;

- as responsabilidades de cada envolvido nas diferentes atividades;

- os formulários a serem utilizados pelos projetos;

- estabelecer quality-gates (qualidade nas etapas) nos principais milestones (marcos) do fluxo dos projetos com as devidas aprovações necessárias para as próximas etapas do projeto;

- desenvolver e aplicar treinamentos englobando toda a nova metodologia para os participantes do processo;

- criar indicadores para cada uma das etapas do fluxo de projetos;

- estabelecer a sistemática de acompanhamento do portfólio de projetos do programa;

- desenvolver uma ferramenta de TI para a gestão e o acompanhamento do portfólio de projetos.

O primeiro ano de atuação do PMO focou a estruturação da metodologia de gerenciamento de projetos e o início do suporte pela área, aos projetos da instituição. Já o ano de 2014 representou a ampliação da atuação do PMO envolvendo a estruturação de programas de treinamentos aos alunos e o auxílio às organizações externas à universidade para a estruturação de novos PMOs.

Foram realizadas entrevistas com o responsável pela implementação e gestão do escritório de projetos e com professores usuários dos processos e metodologias desenvolvidas pela área.

O processo de implantação do PMO na instituição foi conduzido como um projeto gerenciado pelo professor responsável pela área na instituição. Esse professor, além de estudioso do tema, 
tendo feito sua dissertação de mestrado e tese de doutorado neste assunto, possui experiência prática em gestão de projetos em duas organizações de grande porte do segmento industrial.

A decisão de implantação do PMO foi tomada pela pró-reitoria acadêmica da instituição, com o suporte do diretor executivo do programa de pós-graduação em administração e contou com o suporte destes dois sponsors, sendo este um dos principais fatores de sucesso para a implementação.

O modelo de escritório de projetos adotado pela instituição foi o Centro de Excelência em Gerenciamento de Projetos segundo Dinsmore (1998). Este modelo tem as seguintes características:

- funciona como ponto focal da experiência em projetos, mas não assume a responsabilidade direta pelos resultados deles;

- sua tarefa inclui estabelecer e disseminar a metodologia e os processos e transformar os adeptos em profissionais, capacitando-os;

- deve manter abertos os canais de informação entre os projetos e as demais áreas da instituição.

Foram estabelecidos documentos para os processos de iniciação, planejamento, execução, controle e encerramento de projetos. Para os documentos, foi adotada a metodologia do PMI (2013) e desenvolvidos os templates conforme necessidade da instituição.

A nova área integrou-se à estrutura do programa de pós-graduação em administração, ficando sob a responsabilidade de um dos professores da área. Além de suas atribuições como professor em regime de dedicação exclusiva ao programa, esse professor assumiu tal responsabilidade adicionalmente às suas funções.

Faz parte das atribuições do PMO contribuir para a disseminação da metodologia de gerenciamento de projetos dentro da instituição, auxiliar na gestão do conhecimento, no desenvolvimento das competências e metodologia de gerenciamento de projetos na universidade, promover o processo de transferência de conhecimento para a sociedade, e auxiliar a instituição na geração de conhecimento e tecnologia.

O PMO também tem como objetivo apoiar a produção técnica e tecnológica de professores e pesquisadores.

Dentre as várias atividades conduzidas pelo PMO ao longo de 2014, a principal delas consistiu na condução de um treinamento em gestão estratégica e implantação de PMOs.

Este treinamento, em forma de uma disciplina com 32 horas de duração, teve com principais tópicos: gestão de portfólio de projetos, maturidade em gestão de projetos, modelos de PMOs e 
implantação de PMOs. Ao todo foram 20 alunos do curso de pós-graduação latu senso Master in Project Management (Especialista em Gestão de Projetos) que participaram deste treinamento.

O treinamento tinha os seguintes objetivos:

- preparar profissionais do mercado para estruturar um PMO ou exercerem a função de executivo de projetos ou PMO, com o objetivo de gerenciar múltiplos projetos envolvendo ambientes de alta competitividade e complexidade;

- preparar profissionais para atuarem como consultores, trabalhando na implementação de PMOs em organizações de portes pequeno e médio ou em departamento de grandes organizações;

- aprimorar conhecimentos e competências para tratar de forma integrada os aspectos estratégicos do alinhamento do portfólio de projetos das organizações, avaliando conjuntamente a maturidade da organização em gerenciamento de projetos.

Foi solicitada aos alunos a elaboração de um trabalho final individual ou em grupo, que consistia em preparar o plano de implantação de um PMO em uma organização de livre escolha do aluno (se o trabalho fosse individual) ou de seu grupo. Este planejamento deveria incluir todas as etapas do processo de estruturação de um PMO, incluindo seu alinhamento estratégico junto à organização, a discussão dos fatores críticos de sucesso, a medição da maturidade da organização em gestão de projetos, a definição das funções do PMO, a escolha de seu modelo mais adequado e de seus futuros indicadores de desempenho, a montagem da estrutura de governança do PMO e a programação das atividades iniciais do PMO, incluindo os treinamentos necessários de serem ministrados para a organização.

\section{Informações Obtidas com os Objetivos, as Proposições e a Implantação do PMO}

A seguir são apresentadas as correlações entre as informações coletadas na pesquisa e a revisão de literatura realizada como parte deste trabalho.

\section{Como ocorre o processo de implantação do PMO em IES.}

A primeira fase da implantação de um PMO é a decisão de tê-lo ou não na organização (Abe e Carvalho, 2005). Valeriano (2005) descreve que a instalação de um PMO deve ser objeto de um 
projeto específico. Para Desouza e Evaristo (2006), a identificação do motivo que fundamenta a existência do PMO, a elaboração do seu planejamento estratégico e a definição de métricas para avaliação de desempenho são fatores críticos de sucesso na implantação do PMO. Dessa forma, observou-se a necessidade de um PMO, pois a IES possui alguns projetos que precisavam de apoio. Outro fator relevante para a implantação era a experiência do responsável pelo PMO, que além de professor de um dos mestrados em administração da instituição, também implantou PMO em organizações de grande porte.

\section{Alinhamento estratégico entre o PMO e a IES}

Este é um fator decisivo de sucesso na introdução de uma estrutura de gestão de projetos (Aubry et al., 2010; Carvalho e Rabechini,Jr., 2005; Desouza e Evaristo, 2006; Kerzner, 2011). A alta administração precisa reconhecer o valor e o impacto do gerenciamento formal de projetos no desempenho institucional (Anselmo e Maximiano, 2006; Carvalho e Rabechini,Jr., 2005; Desouza e Evaristo, 2006). O PMO teve total apoio da direção da IES, pois uma de suas estratégias é o reconhecimento da excelência em seus projetos.

\section{Características e atribuições do PMO na IES}

Segundo Eersen, Henriksen e Aarseth (2007), Dai e Wells (2004), Hobbs e Aubry (2008) e Valeriano (2005) o PMO é responsável pelo desenvolvimento de um conjunto de normas e regras que representam a metodologia própria da organização para a gestão de seus projetos com o intuito de padronizar os processos. Foram criados templates para os processos e definida a sistemática de padronização quanto ao apoio do PMO para os eventos e projetos organizados pela IES, além de treinamento para todos os envolvidos.

\section{Disseminação da metodologia de gerenciamento de projetos}

O PMO, do ponto de vista organizacional, pode ser visto como uma ferramenta de apoio à gestão do conhecimento nas instituições (Kawahara e Jussani, 2013) para a seleção, priorização e integração dos projetos para alcance dos objetivos organizacionais, desenvolvimento de metodologia e armazenamento de lições aprendidas (Crawford, 2002; PMI, 2013). Pôde-se observar 
que o fato da IES possuir um curso de pós-graduação stricto sensu na área de gestão de projetos ajudou na disseminação da metodologia e principalmente pela experiência do responsável pelo PMO, aplicando treinamentos sobre a metodologia.

\section{Caracterização do PMO da IES estudada}

A função principal do PMO é apoiar os projetos desenvolvidos no programa stricto sensu e à organização de um simpósio internacional. Desta forma, foi corroborado que o PMO na IES trafega entre o $2^{\circ}$ e $3^{\circ}$ nível, segundo a classificação de Dinsmore (1998), ou seja:

- $2^{\circ}$ Nível - Escritório de Suporte aos Projetos (PSO) - Apoio técnico e administrativo às mudanças de escopo e ao gerenciamento de custos, responsabilidade pelo sucesso é do gerente de projeto.

- $3^{\circ}$ Nível - Centro de Excelência de Gerenciamento de Projetos (PMCOE) - Disseminação das práticas de gestão de projetos, capacitação dos membros, conversão dos incrédulos, não assume a responsabilidade pelo sucesso do projeto.

No caso do PMO na IES a responsabilidade do sucesso pelos projetos é compartilhada.

\section{Plano de Implantação de PMOs em IES}

Com base no caso estudado é proposto um modelo para implantação de PMOs em IES. Este plano é composto por 10 documentos:

- Termo de Abertura;

- Estrutura Analítica do Projeto;

- Cronograma;

- Orçamento;

- Organograma;

- Matriz de Responsabilidades;

- Matriz de Comunicações;

- Indicadores de Qualidade;

- Relação de Aquisições;

- Matriz de Riscos. 


\section{Conclusões}

Para responder à questão de pesquisa apresentada: Como implantar um PMOs em IES? Foi necessário envolvimento com a literatura que considera a gestão de projetos, os PMOs, as organizações privadas, a estratégia e o desempenho organizacional, além da identificação de uma instituição que não só permitisse o desenvolvimento da pesquisa em uma das linhas de pesquisa de interesse dos pesquisadores, mas que também possibilita a integração com outras áreas de pesquisa, estes são critérios de destaque presentes neste trabalho.

Chegar à resposta do problema de pesquisa demandou o entendimento e a apresentação sobre como ocorre o processo de implantação do PMO nas organizações privadas, sobre o alinhamento estratégico entre as duas estruturas, sobre as particularidades dos projetos e as características e atribuições do PMO também nas instituições de ensino superior, para, enfim, avaliar como o processo de implantação ocorreu.

Vale ressaltar que a implantação do PMO-IES corrobora a teoria apresentada, na medida em que: originou de uma decisão institucional (Abe e Carvalho, 2005) e foi resultado de um projeto específico, embasado pelos objetivos e necessidades da organização (Valeriano, 2005).

Conforme revisado no referencial teórico, os PMOs possuem características e atribuições, das quais o estabelecimento de um conjunto de normas e regras para a gestão de projetos (Eersen et al., 2007; Daí e Wells, 2004; Hobbs e Aubry, 2008; Valeriano, 2005), o investimento na equipe (Hobbs e Aubry, 2008; Valeriano, 2005), a atenção às lições aprendidas (Daí e Wells, 2004; Valeriano, 2005) e a promoção da inovação (Hobbs e Aubry, 2008; Aubry et al., 2010) merecem especial destaque.

Dessa maneira, vale destacar que todos os processos citados pelos autores acima foram realizados pelo responsável pela implantação do PMO, inclusive cabe ressaltar, conforme descrito em seções anteriores, o treinamento ministrado em forma de consultoria pelo PMO para a criação de um plano de implantação para diferentes organizações privadas. Por meio desse treinamento foi possível corroborar junto à literatura e à prática de gestão de projetos e principalmente elaborar o plano de implantação de PMO em IES conforme questão de pesquisa deste trabalho. 
Na Figura 5 foi elaborada de forma detalhada a sequência do plano de implantação do PMO em IES seguindo também os mesmos templates citados anteriormente.

\begin{tabular}{|c|c|c|}
\hline 10 Passo & \multicolumn{2}{|c|}{ Área de atuação da empresa } \\
\hline 20 Passo & Avaliação Estratégica & to da Instituição na Matriz Volume Variedade \\
\hline 30 Passo & \multicolumn{2}{|c|}{ Organograma da Empresa } \\
\hline 40 Passo & \multicolumn{2}{|c|}{ Tipo de Estrutura e PMOs atuais } \\
\hline 5o Passo & \multicolumn{2}{|c|}{ Tipo de Estrutura e PMOs propostos } \\
\hline 60 Passo & Avaliação de Maturidade PMMM & Avaliação de Maturidade OPM3 \\
\hline 70 Passo & Plano Operacional & Utilização dos TEMPLATES \\
\hline
\end{tabular}

Figura 5. Classificações dos tipos de escritórios de projetos Fonte: Elaborada pelos autores

A partir de toda a análise discorrida por este artigo, a resposta ao problema de pesquisa é que é possível implantar PMO em IES por meio do plano de implantação seguindo os passos citados na Figura 5 e essencialmente operacionalizar os documentos apresentados na seção anterior.

A opção metodológica adotada neste trabalho, estudo de caso único, não permite analisar outras instituições e compará-las. Yin (2010) destaca que o estudo de caso único não permite uma amplitude de casos, ficando assim limitado ao estudo de um único caso. Desta forma, destaca-se a restrição quanto ao universo pesquisado, uma vez que foi considerada uma única unidade de análise. Torna-se necessário esclarecer que devido à profundidade do estudo de caso único e ao tempo destinado para o trabalho, foi possível certa profundidade neste estudo. Mesmo com a impossibilidade quanto à generalização, o caso apresentado consta como um exemplo de 
experiência que pode ser utilizado como referência para outras instituições e também como fonte para novas pesquisas acadêmicas.

Outra limitação do estudo está relacionada à inabilidade também temporal, além do limitador físico, para a exploração de outros temas que trariam contribuição para as análises abordadas. Dois potenciais conteúdos enriqueceriam ainda mais este estudo: um deles seria a medição do impacto da implantação do PMO na IES e como ocorreria o processo de transferência de conhecimento gerado pelo escritório tanto para a instituição quanto para a sociedade.

Seguindo, então, as próprias limitações do presente estudo, sugere-se que próximos trabalhos analisem os impactos do PMO nas organizações à luz do nível de maturidade do escritório de gerenciamento de projetos. Acredita-se que, quanto maior o nível de maturidade, maior será o impacto.

Fazer também uma relação do nível de alinhamento estratégico entre o PMO e a instituição e do resultado obtido pela estrutura pode representar outra contribuição para a literatura e para a prática organizacional. Sabe-se que o alinhamento estratégico é relevante e essencial para o sucesso, mas quais resultados o PMO promove, se alinhado em diferentes níveis à instituição, pode ser uma interessante pergunta a responder.

Como terceira e última sugestão, propõe-se um novo estudo a partir da mesma unidade analisada com o objetivo de contrastar resultados futuros e acompanhar bem como apresentar para a literatura a sua evolução. Consistiria em uma importante contribuição, tanto para a ciência quanto para as organizações, a verificação a médio e longo prazo de quais resultados o PMO tem obtido após a sua implantação ainda nos níveis iniciais de maturidade.

Perceber se os impactos esperados foram alcançados e se as expectativas apresentadas pela presente pesquisa foram atingidas em maior ou menor grau do que o esperado direcionaria novas ações de implantação de PMOs, garantindo ou não a segurança para os gestores no desenvolvimento de uma iniciativa como o da instituição estudada neste trabalho.

\section{Referências}

Abe, C. K. \& Carvalho, M. M. (2005). Fatores críticos para a implantação do PMO: um estudo de caso. Gepros - Gestão da Produção, Operações e Sistemas. Ano 1, n. 3.

Anselmo, J. L., Maximiano, A. C. A. (2006, out./nov./dez.). PMO: um estudo de caso. Revista Administração. São Paulo, vol.41, n.4, pp.394-403.

Aubry, M. \& Hobbs, B. (2008). The Project Management Office (PMO): A quest for understandieing. 
Aubry, M., Muller, R., Hobbs, B. \& Blomquist, T. (2010). Project Management Offices in Transition. International Journal of Project Management, 28(8), 766-778. doi:10.1016/j.ijproman.2010.05.006.

Barbalho, S. C. M.; Amaral, D. P.; Kernbichler, T. S.; Richter, E. H. \& Torres, L. (2009, jul./set.). Rompendo obstáculos para a implantação de PMO em empresa de base tecnológica. Gestão e Produção. São Carlos, vol. 16, n. 3, pp. 435-449.

Brasil (1996). A Lei no 9.394, de 20 de dezembro de 1996. Lei de Diretrizes e Bases da Educação Nacional (LDB). Art. 43-57.

Capes. Plataforma Lattes. Recuperado em 20 de dezembro, 2014, de http://lattes.cnpq.br/.

Capes/MEC. Diretoria de Avaliação - DAV. (2013, fev.) Coleta de Dados 12.0 - Manual do Usuário.

Carvalho, K. E. M. (2013). Impactos da implantação do PMO no desempenho da organização pública: o caso de uma instituição de ensino superior. Dissertação de mestrado profissional em administração: Gestão de Projetos. Universidade Nove de Julho. São Paulo, SP, Brasil. Recuperado em 20 de dezembro, 2014. http://www4.uninove.br/tedeSimplificado/tde_busca/processaPesquisa.php?listaDetalhes\%5B\%5D=533epro cessar=Processar

Carvalho, M. M. \& Rabechini Jr. R (2005). Construindo competências e, gerenciamento de projetos: teoria e casos. São Paulo: Editora Atlas.

(2011). Fundamentos em gestão de projetos: construindo competências para gerenciar projetos (3a. ed.), São Paulo: Editora Atlas.

Coelho, T. P.; Dubke, A. F. \& Nascimento, F. C. G. (2010, jul./dez.). A transferência de conhecimento na implantação de um PMO. Revista Gestão e Projetos - GeP, São Paulo, vol. 1, n. 2, pp. 147-161.

Crawford, J. K. (2002). The Strategic Project Office - A Guide to Improving Organizational Performance. New York: Marcel Dekker. Recuperado em 21 de abril, 2014, de http://books.google.com.br/books?id=Pay44GuJBigCeprintsec=frontcoveredq=The+Strategic+Project+Offic eehl=pt-

BResa $=$ Xeei=TpRVU7nbJMfUsATR0oD4Dweved=0CDYQ6AEwAQ\#v=onepageeq $=$ The $\% 20$ Strategic $\% 20$ Project\%20Officeef=false

Dai, C. X. \& Wells, W. G. (2004). An Exploration of Project Management Office Features e Their Relationship to Project Performance. International Journal of Project Management, 22(7), 523-532.

Desouza, K. C. \& Evaristo, J. R. (2006). Project Management Offices: a case of knowledge-based archetypes. International Journal of Information Management, 414-423

Dinsmore, P. C. (1998). Gerência de programas e projetos. São Paulo: PINI Editora

Dinsmore, P. C. \& Cabanis-Brewin, J. (2006). The AMA Handebook of Project Management. (second edition. Amacom American Management Association.

Eersen, B.; Henriksen, B. \& Aarseth, W. (2007, april). Benchmarketing of project management office establishment: Journal of Management in Engineering. 97-104. 
Garnica, L. A. \& Torkomian, A. L. V. (2009, out/dez.). Gestão de tecnologia em universidades: uma análise do patenteamento e dos fatores de dificuldade e de apoio à transferência de tecnologia no Estado de São Paulo. Revista Gestão e Produção, São Carlos, vol. 16, n. 4, pp. 624-638.

Gil, A. C. (2008). Métodos e técnicas de pesquisa social (6a. ed.) São Paulo: Atlas, pp. 57-58.

Kawahara, K. \& Jussani, A. C. (2013, novembro 7 e 8). Fatores Críticos de Sucesso na implantação de um PMO: estudo de caso em uma empresa de consultoria em tecnologia da informação. II Simpósio Internacional e Gestão de Projetos (II Singep) Simpósio Internacional e Inovação e Sustentabilidade (I S2IS). Anais do II Singep e I S2IS - São Paulo - SP - Brasil.

Kerzner, H. (2011). Gerenciamento de Projetos: uma abordagem sistêmica para planejamento, programação e controle (11a. ed.). São Paulo: Editora Blucher.

Kniess, C. T. \& Moutinho, J. A. (2012, maio/ago.). Contribuições de um PMO em um laboratório de P\&D de uma universidade pública. Revista Gestão e Projetos - GeP, São Paulo, vol. 3, n. 2, pp. 260-271; 282-293.

Marconi, M. A. \& Lakatos, E. M. (2005). Fundamentos de metodologia científica (6a. ed.). São Paulo: Atlas, pp. 165-166.

Moraes, R. \& Stal, E. (1994). Interação universidade-empresa no Brasil. RAE - Revista de Administração de Empresas, São Paulo, vol. 34, n. 4, pp. 98-112.

Patah, L. A. \& Carvalho, M. M. (2003). O Processo de Implantação de um Project Management Office. In: Seminário Gestão de Projetos 2003 Sucesu-SP. São Paulo.

Patah, L. A. (2004). Alinhamento estratégico de estrutura organizacional de projetos: uma análise de múltiplos casos. Dissertação de mestrado, Universidade de São Paulo, São Paulo, SP, Brasil.

Project Management Institute (2013). A Guide to the Project Management Body ok knowledge (PMBOK Guide) - Fifth Edition.

Segatto-Mendes, A. P. (2001). Teoria de Agência Aplicada à Análise de Relações entre os Participantes dos Processos de Cooperação Tecnológica Universidade-Empresa. Tese de doutorado. Faculdade de Economia, Administração e Contabilidade, USP, São Paulo, SP, Brasil. Disponível: http://www.teses.usp.br/teses/disponiveis/12/12139/tde-24012002-114443

Siegel, D. S.; Waldman, D. A.; Atwater, L. E. \& Link, A. N. (2004). Toward a model of the effective transfer of scientific knowledge from academicians to practitioners: qualitative evidence from the commercialization of university technologies. Journal of Engineering e Technology Management JET-M. doi:10.1016/j.jengtecman.2003.12.006

Valeriano, D. (2005). Moderno Gerenciamento de Projetos. São Paulo: Prentice Hall/Pearson 2011 2009, pp. $75-106$.

Yin, R. K. (2010, pp. 38-41). Estudo de Caso: Planejamento e Métodos (4a. ed.). Porto Alegre: Editora Bookman. 